\title{
CMEARTICLE
}

\section{Clinics in diagnostic imaging (202)}

Jeremy Jia Hong $\underline{\mathrm{Lam}}^{1}$, MBBS, Sandeep Halagatti Venkatesh${ }^{2}$, MBBS, FRCR, Chi Long $\underline{H o}^{2}$, MD, FRCR, Bak Siew Steven Wong' ${ }^{2}$, MBChB, MMed
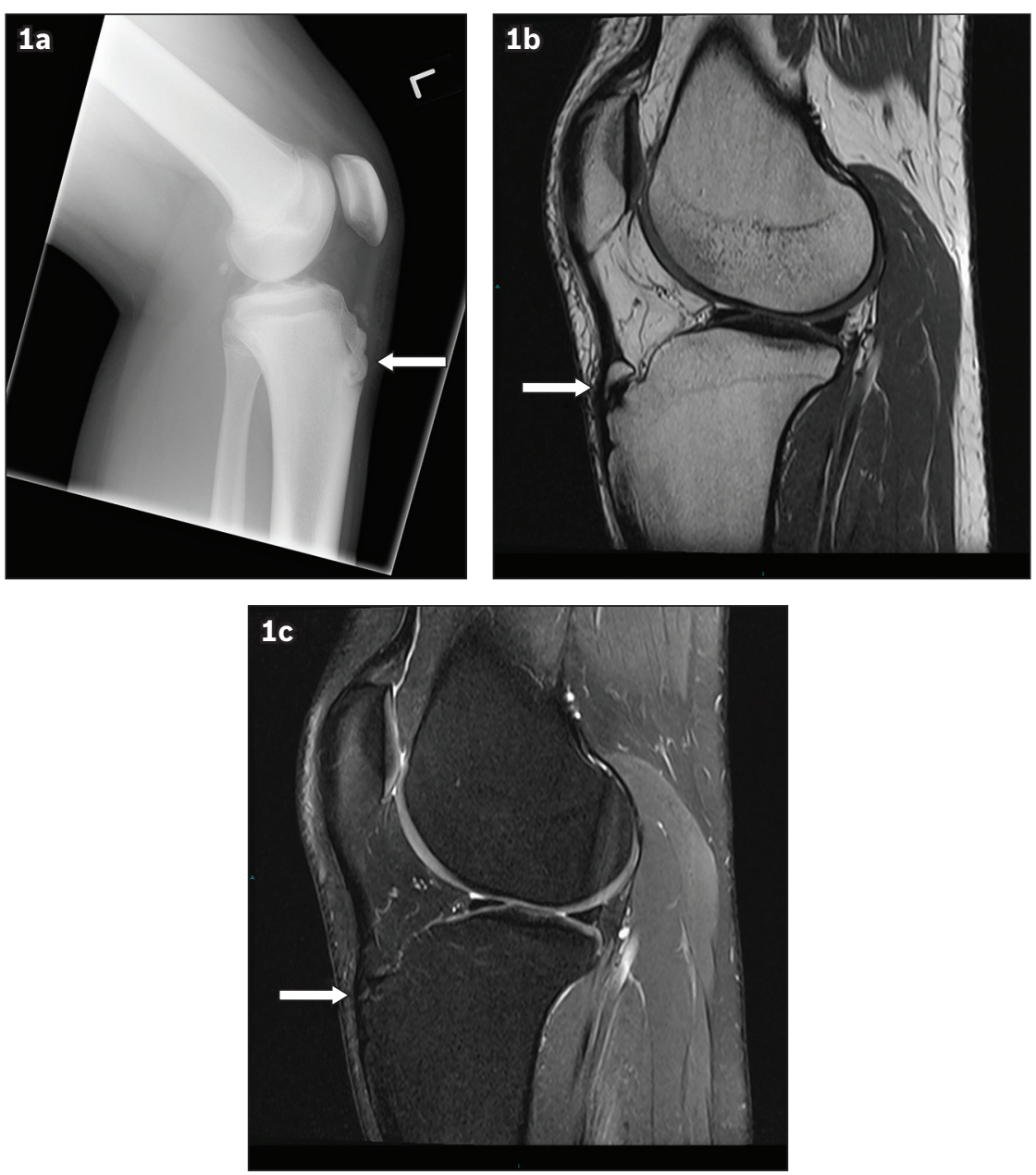

Fig. 1 (a) Lateral radiograph; (b) sagittal proton density-weighted turbo spin echo sequence; and (c) sagittal proton density-weighted turbo spin echo fat-saturated sequence of the left knee.

\section{CASE PRESENTATION}

A 14-year-old boy presented with anterior left knee pain of two weeks' duration with no history of trauma. The knee pain was aggravated by exercise and relieved by rest. On physical examination, there was focal tenderness at the left tibial tuberosity, and the pain was reproducible on forced extension of the knee. The rest of the knee examination did not reveal any joint instability. Radiography of the knee was performed (Fig. 1a). What does the image show and what is the diagnosis? As the pain persisted, magnetic resonance (MR) imaging of the knee was subsequently performed (Figs. $1 \mathrm{~b} \& \mathrm{c}$ ). What do the images show? 


\section{IMAGE INTERPRETATION}

Radiograph of the left knee (Fig. 1a) shows bony irregularity with fragmentation at the tibial tubercle. A small, well-corticated bony fragment superior to the tibial tuberosity is noted (arrow in Fig. 1a). There is mild soft tissue swelling at the expected patellar tendon insertion site but no significant joint effusion.

Subsequent MR imaging of the knee demonstrates a small ossicle in the distal pre-insertional part of the left patellar tendon, which shows well-defined corticated margins and no identifiable marrow oedema (Fig. 1b). The adjacent left tibial tuberosity shows mild marrow oedema just deep to the articulation with the ossicle (Fig. 1c). The rest of the patellar tendon is normal in thickness and signal characteristics.

\section{DIAGNOSIS}

Osgood-Schlatter disease (OSD).

\section{CLINICAL COURSE}

The patient was treated conservatively with administration of a short course of oral analgesics along with rest and modification of exercise. The symptoms subsequently resolved with no further functional impairment or pain.

\section{DISCUSSION}

OSD is a form of traction osteochondritis involving the tibial tubercle. It was first described in 1903 by Dr Robert Osgood and Dr Carl Schlatter separately, within the same year..$^{(1,2)}$ OSD is characterised clinically by the presence of pain and swelling of the tibial tuberosity in the adolescent patient. ${ }^{(3)}$ It is widely accepted as a traction apophysitis involving the tibial tubercle and the distal aspect of the patellar tendon. There is chronic and repetitive injury to the distal patellar tendon and avulsions of the cartilaginous attachment of the patellar tendon to the secondary ossification centre of the tibial tubercle. Foci of heterotopic ossification may occur when the avulsed cartilage fragments ossify. ${ }^{(3)}$

OSD typically occurs in the adolescent due to recurrent avulsion and microtrauma of the developing tibial tuberosity. It occurs more commonly in males aged $12-15$ years and earlier in females aged 8-12 years due to the earlier onset of the pubertal growth spurt, and may manifest bilaterally in $20 \%-30 \%$ of patients. ${ }^{(4)}$ Higher incidences of OSD are observed in those who are active in sports compared to those who are inactive, at $21 \%$ and $4.5 \%$, respectively. ${ }^{(3)} \mathrm{A}$ review of 794 published cases in the literature revealed that $72 \%$ of all cases of OSD are male. ${ }^{(5)}$ The male predominance has been attributed to greater participation in sports and faster skeletal growth in the male adolescent.

Patients with OSD usually present with pain over the anterior aspect of the knee and tenderness with application of pressure on the tibial tubercle. Any activity requiring contraction of the quadriceps mechanism aggravates the pain, which improves with rest. There may be enlargement of the tibial tuberosity, thickening of the patellar tendon or joint swelling. On physical examination, tenderness is elicited on palpation or application of pressure on the tibial tubercle and patellar tendon. As in our patient, pain is reproducible by resisted active extension of the knee. ${ }^{(3)}$

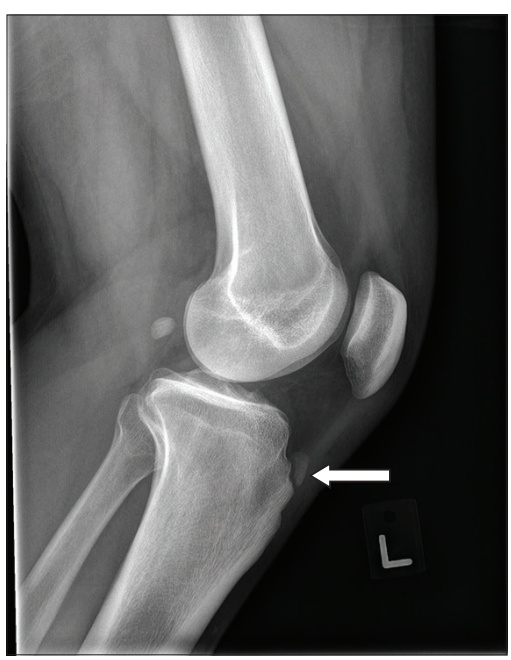

Fig. 2 A 16-year-old boy presented with progressively worsening pain over the left tibial tuberosity. Lateral radiograph of his left knee shows a welldefined ossific fragment at the distal attachment of the patellar (arrow), which is suggestive of Osgood-Schlatter disease.

The diagnosis of OSD is based on clinical examination, with additional radiographic investigations to exclude fractures or bony tumours. ${ }^{(3)}$ Radiographs of the knee may demonstrate fragmentation, increased density, irregularity or enlargement of the tibial tubercle (Fig. 2).

On MR imaging, the normal patellar tendon should be homogeneously low signal on T1-, T2- and proton densityweighted images. The normal thickness of the patellar tendon increases proximally to distally, but should not exceed $7 \mathrm{~mm}$ in thickness. ${ }^{(6)}$ The presence of either focal or diffuse thickening and/ or intermediate T1-weighted and T2-weighted signal may represent pathology. ${ }^{(7)}$ MR imaging in the sagittal plane in patients with OSD may reveal enlargement of distal aspects of the patellar tendon, low signal intensity foci of heterotopic ossification, and irregularity or enlargement of the tibial tuberosity. Distention of the deep infrapatellar bursa may be due to the presence of fluid between the deep surface of the patellar tendon and anterior cortex of the tibia. There may be increased signal on T2-weighted images demonstrating oedema at the tibial tuberosity and tibial epiphysis (Fig. 3). ${ }^{(4,6)}$

Ultrasonography may also demonstrate abnormalities surrounding the patellar tendon attachment such as reactive bursitis, patellar tendon lesions, cartilage swelling and neovascularisation at the tibial tuberosity. ${ }^{(8)}$

Differential diagnoses of OSD include: (a) Sinding-LarsenJohansson (SJS) disease; (b) Hoffa's fat pad impingement syndrome; (c) fracture of the tibial tuberosity; (d) infrapatellar bursitis; and (e) patellar tendonitis.

SJS disease is a common cause of anterior knee pain in active adolescents aged 10-13 years. On physical examination, point tenderness can be elicited by direct pressure over the inferior pole of the patella. Symptoms may also be aggravated by jumping. Like OSD, SJS disease is diagnosed clinically, although radiography should be performed to exclude other pathologies. On imaging, there may be ossific fragments within the proximal patellar tendon or at the inferior pole of the patella, which may show oedema, as evidenced by high signal on fluid-sensitive MR sequences 

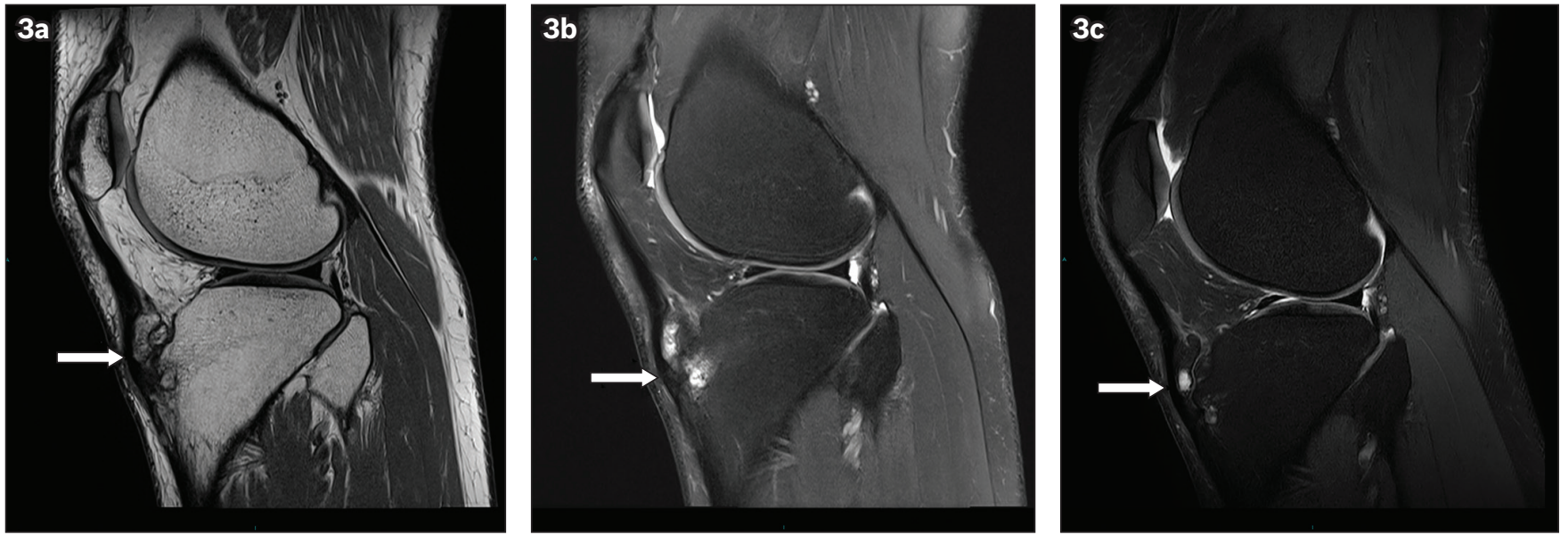

Fig. 3 MR imaging of the left knee was performed for a patient with known Osgood-Schlatter disease. (a) Sagittal proton density-weighted turbo spin echo sequence shows bony fragmentation at the tibial tuberosity adjacent to the distal insertion site of the patellar ligament (arrow). (b) Sagittal proton density-weighted turbo spin echo fat-saturated sequence shows bone oedema and cyst formation at the tibial tuberosity (arrow). (c) Sagittal proton density-weighted turbo spin echo fat-saturated sequence done two years later shows resolution of marrow oedema but interval development of intraosseous cystic changes within a bony fragment (arrow) adjacent to the tibial tuberosity.
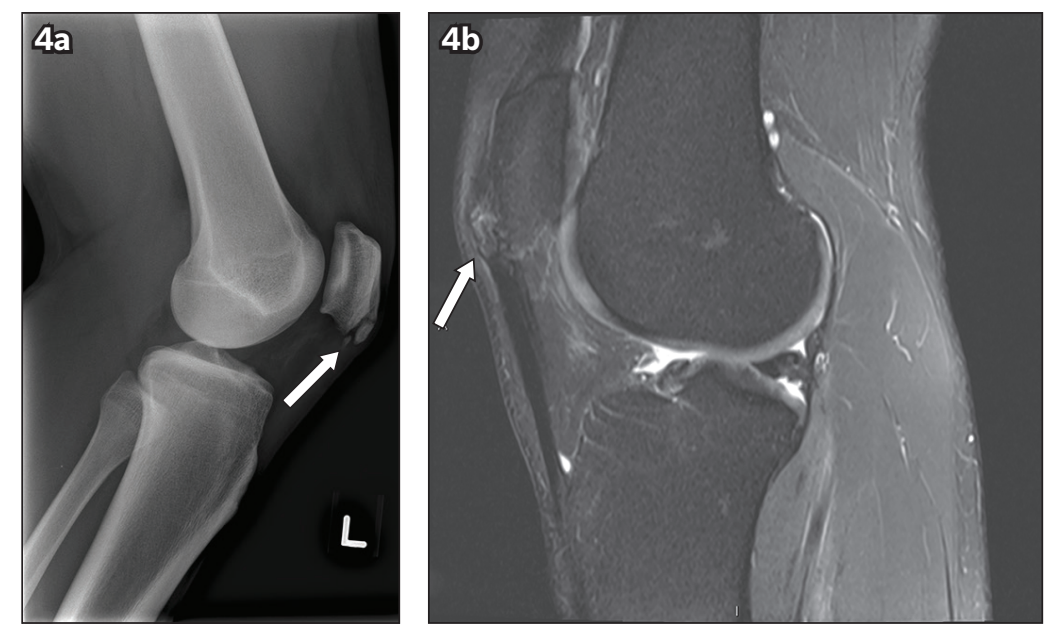

Fig. 4 Radiography and MR imaging were performed for a patient with Sinding-Larsen-Johansson disease. (a) Lateral radiograph shows ossification within the proximal patellar tendon adjacent to the inferior pole of the patella (arrow). No donor site is seen at the inferior pole of the patella. (b) Sagittal proton density-weighted turbo fat-saturated sequence of the same patient shows ossific fragments at the inferior pole of the patella (arrow). Minimal oedema is noted within the ossific fragments and adjacent patella.

(Fig. 4). It is a self-limiting condition that responds to modification of activities and simple analgesics. ${ }^{(9)}$

Hoffa's fat pad impingement syndrome involves impingement of the infrapatellar fat pad. An initial injury or repetitive trauma to the infrapatellar fat pad results in haemorrhage. This leads to inflammation and hypertrophy, predisposing the fat pad to impingement between the femur and tibia (Fig. 5). In the absence of injury, impingement of the fat pad may also occur due to narrowing of the joint space from any cause. ${ }^{(10)}$ In fractures of the tibial tuberosity, there is a history of preceding trauma with sudden onset of pain. On radiographs, a fracture line will be evident without fragmentation of the tibial tuberosity ${ }^{(11)}$ (Fig. 6). MR imaging will clearly show the marrow oedema related to the fracture.

Clinically, it is difficult to differentiate infrapatellar bursitis from OSD, as the pain localises to or near the patellar tendon attachment to the tibial tuberosity. On MR imaging, the tibial tuberosity is normal in appearance but may show an infrapatellar fluid collection. ${ }^{(11)}$ Large collections may be visible as increased soft tissue density on plain radiographs (Fig. 7).

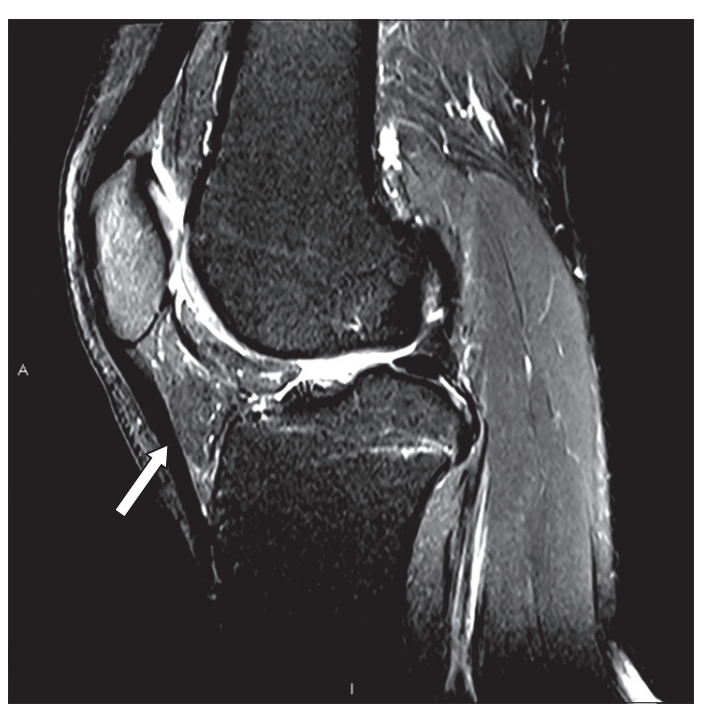

Fig. 5 A patient presented with anterior knee pain. MR image shows subtle hypertrophy of the Hoffa's fat pad (arrow), which predisposes it to impingement between the femur and tibia. In the appropriate clinical setting, this may be related to impingement of Hoffa's fat pad. 


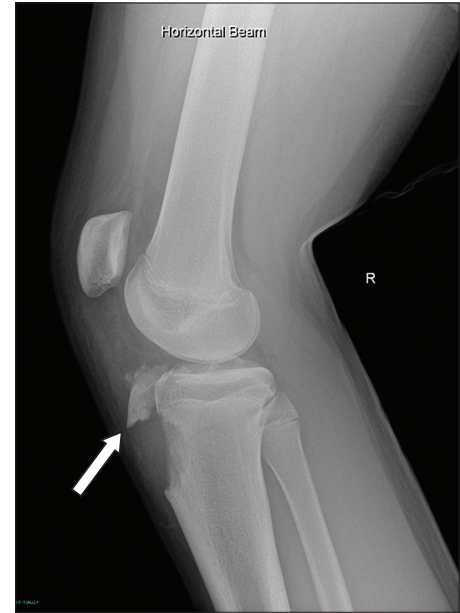

Fig. 6 A patient presented with a history of trauma and anterior knee pain Lateral knee radiograph shows displaced fracture fragments (arrow) at the anterior aspect of the knee with a donor site in the proximal tibia at the expected site of the tibial tuberosity.

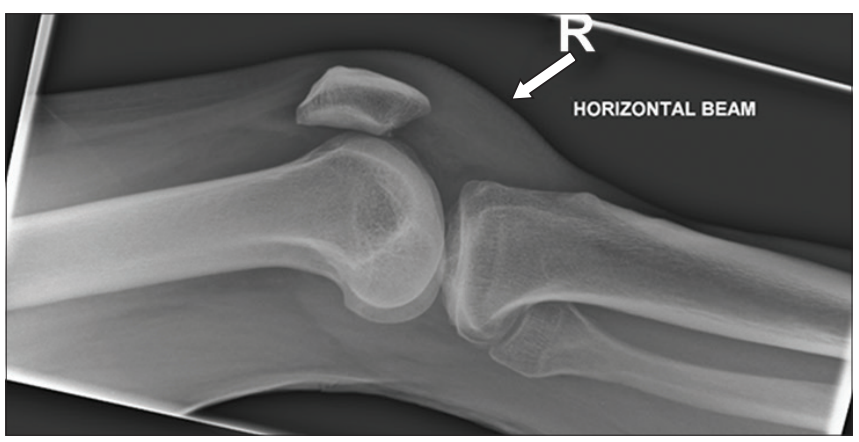

Fig. 7 A patient with infrapatellar bursitis presented with localised knee pain anterior to the tibial tubercle. The lateral radiograph shows increased soft tissue density in the infrapatellar region (arrow), likely representing an inflamed/distended infrapatellar bursa.

Patellar tendonitis may occur as a complication of OSD. Radiographs may be normal or show soft tissue swelling. On MR imaging, the tibial tuberosity appears normal and there may be increased signal in the patellar tendon (Fig. 8). ${ }^{(11}$

Conservative measures consisting of local application of ice packs and oral administration of analgesics comprise the mainstay of therapy. Modification of exercise is suggested with avoidance of strenuous activities. Strengthening and stretching exercises may also help. Protective pads that are worn over the tibial tubercle may help to avoid direct trauma to the tibia tubercle, although cast immobilisation is not advised, as wasting of the quadriceps muscle may occur. Surgical management may be considered in patients when conservative measures have failed and after fusion of the tibial growth plate. ${ }^{(3)}$ Surgical management is rarely required for unresolved OSD. In cases where there are persistent symptoms after skeletal maturity, surgical treatment may be advocated with good outcomes and without long-term deleterious effects. ${ }^{(12)}$ One study showed promising results for hyperosmolar dextrose injection together with lidocaine over the apophysis and patellar tendon origin, demonstrating subsequent improvement of symptoms, although more research is needed before this procedure can be routinely recommended. ${ }^{(13)}$ In general, OSD tends to be self-limiting with resolution of symptoms in more than $90 \%$ of patients and good overall prognosis with non-operative treatment.
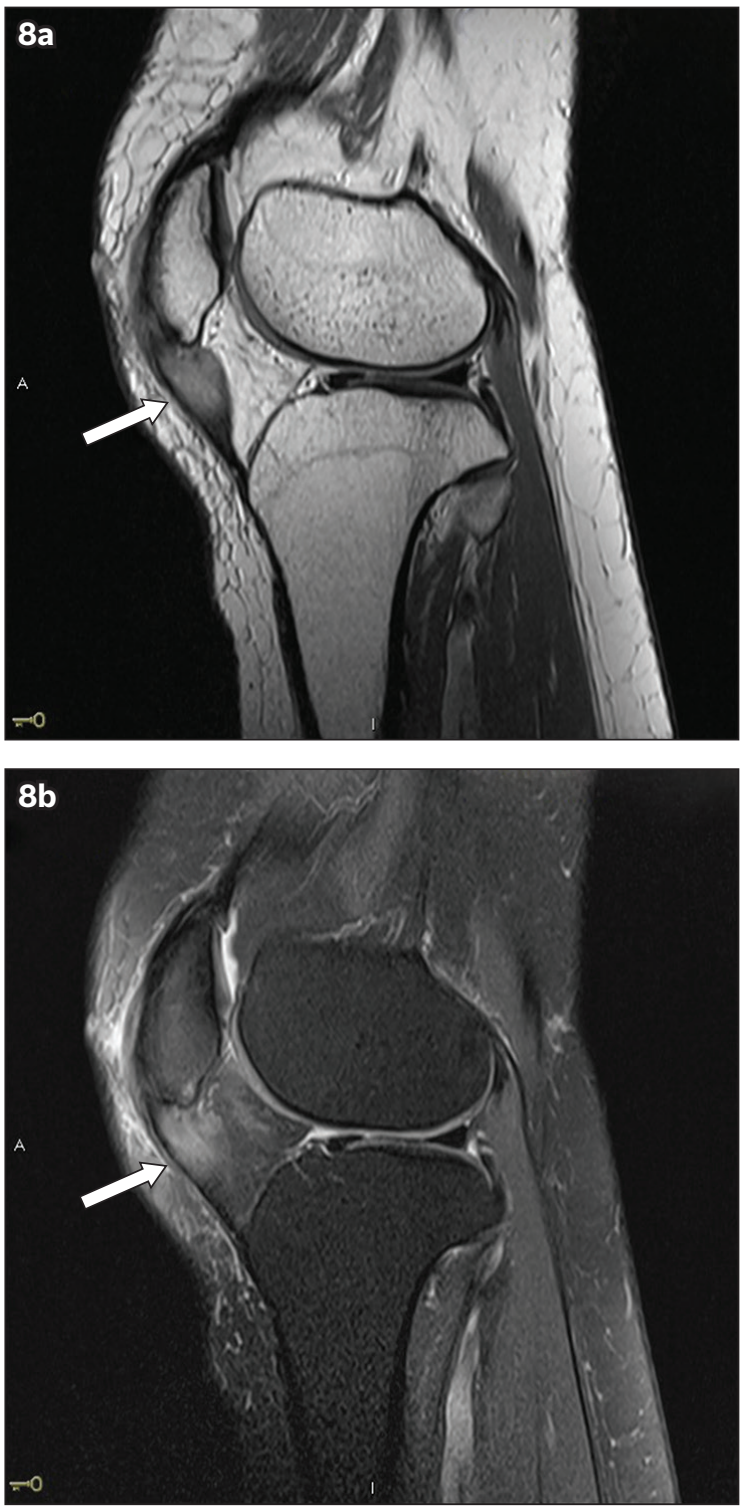

Fig. 8 A patient presented with anterior knee pain after minor trauma. (a) Sagittal proton density-weighted turbo spin echo sequence shows thickening and increased signal (arrow) in the proximal patellar tendon. (b) Sagittal proton density-weighted turbo spin echo fat-saturated sequence of the same patient shows increased signal (arrow) in the proximal patellar tendon and surrounding infrapatellar fat pad, representing oedema.

ABSTRACT A 14-year-old boy presented with left anterior knee pain, which was aggravated by exercise and relieved by rest. On clinical examination, there was tenderness at the tibial tuberosity with reproducible pain on resisted active extension of the knee. Radiographs showed heterotopic ossification of the patellar tendon with irregularity and fragmentation of the tibial tubercle. Clinical and radiological findings were consistent with Osgood-Schlatter disease, which is a traction apophysitis of the tibial tubercle commonly occurring in adolescents. The clinical presentation and imaging features are discussed.

Keywords: diagnostic imaging, education, knee, Osgood-Schlatter disease, osteochondrosis 


\section{REFERENCES}

1. Osgood R. Lesions of the tibial tubercle occurring during adolescence. 1903 Clin Orthop Relat Res 1993; (286):4-9.

2. Schlatter C. Injury of the bony tuberosity of the proximal tibial epiphysis. Beitr Klin Chir Tubing 1903; 38:874-8. German.

3. Gholve PA, Scher DM, Khakharia S, Widmann RF, Green DW. Osgood Schlatter syndrome. Curr Opin Pediatr 2007; 19:44-50.

4. Bedoya MA, Jaramillo D, Chauvin NA. Overuse injuries in children. Top Magn Reson Imaging 2015; 24:67-81.

5. Antich TJ, Lombardo SJ. Clinical presentation of Osgood-Schlatter disease in the adolescent population. J Orthop Sports Phys Ther 1985; 7:1-4.

6. O'Keeffe SA, Hogan BA, Eustace SJ, Kavanagh EC. Overuse injuries of the knee. Magn Reson Imaging Clin N Am 2009; 17:725-39.
7. Morrison WB, Major N. The knee. Top Magn Reson Imaging 2015; 24:193-203.

8. Suzue N, Matsuura T, Iwame T, et al. State-of-the-art ultrasonographic findings in lower extremity sports injuries. J Med Invest 2015; 62:109-13.

9. Atanda A Jr, Shah SA, O'Brien K. Osteochondrosis: common causes of pain in growing bones. Am Fam Physician 2011; 83:285-91.

10. Jacobson JA, Lenchik L, Ruhoy MK, Schweitzer ME, Resnick D. MR imaging of the infrapatellar fat pad of Hoffa. Radiographics 1997; 17:675-91.

11. Vaishya R, Azizi AT, Agarwal AK, Vijay V. Apophysitis of the tibial tuberosity (Osgood-Schlatter disease): a review. Cureus 2016; 8:e780.

12. Pihlajamäki H, Visuri T. Long-term outcome after surgical treatment of unresolved Osgood-Schlatter disease in young men: surgical technique. J Bone Joint Surg Am 2010; 92 Suppl 1 Pt 2:258-64.

13. Topol G, Podesta L, Reeves K, et al. Hyperosmolar dextrose injection for recalcitrant Osgood-Schlatter disease. Pediatrics 2011; 128:e1121-8. 


\section{SINGAPORE MEDICAL COUNCIL CATEGORY 3B CME PROGRAMME}

\section{(Code SMJ 201912A)}

Question 1. Regarding Osgood-Schlatter disease:

(a) It is a chronic apophysitis of the tibial tubercle.

(b) It is a traction apophysitis involving the inferior pole of the patella.

(c) It is a fracture of the tibial tuberosity.

(d) There is rupture of the patellar tendon.

Question 2. Regarding imaging of the knee:

(a) The patellar tendon decreases in thickness from its proximal to distal extent.

(b) The patellar tendon typically does not exceed $7 \mathrm{~mm}$ in thickness.

(c) On magnetic resonance (MR) imaging, the normal patellar tendon demonstrates heterogeneous signal intensity on T1-weighted, T2-weighted and proton density-weighted images.

(d) On MR imaging, the normal patellar tendon demonstrates low signal intensity on T1-weighted, T2-weighted and proton density-weighted images.

Question 3. Findings of Osgood-Schlatter disease on a radiograph may include:

(a) Irregularity of the tibial tubercle.

(b) Decreased density of the tibial tubercle.

(c) Fragmentation of the tibial tubercle.

(d) Enlargement of the tibial tubercle.

Question 4. Findings of Osgood-Schlatter disease on MR imaging may include:

(a) Enlargement of the distal patellar tendon.

(b) Low signal intensity heterotopic ossification.

(c) Distention of the infrapatellar bursa.

(d) Increased signal on T2-weighted images demonstrating oedema at the tibial tuberosity and tibial epiphysis.

Question 5. The management of Osgood-Schlatter disease includes:

(a) Corticosteroid injections.

(b) Surgical management when conservative measures fail, after fusion of the proximal tibial growth plate.

(c) Modification of physical activity.

(d) Wearing of a protective pad to prevent trauma to the tibial tubercle.

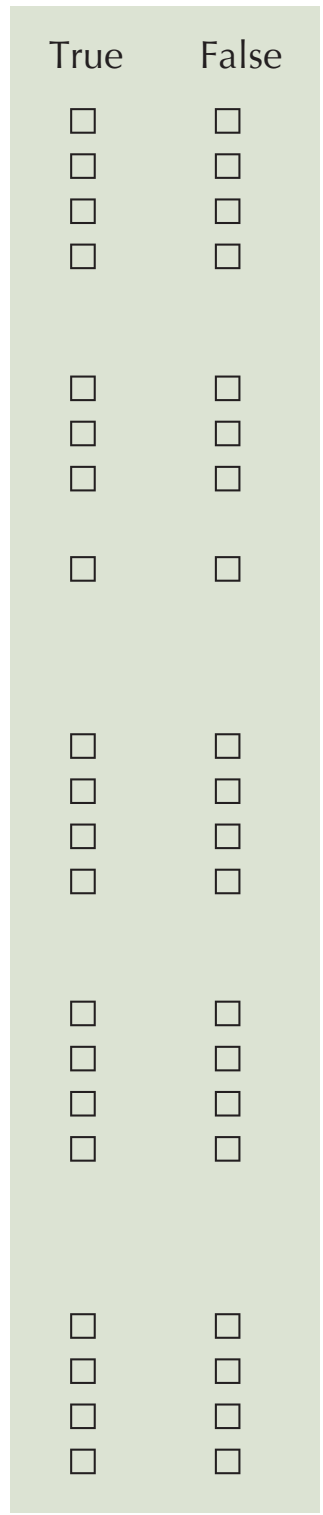

\section{Doctor's particulars:}

Name in full:

MCR no.:

Specialty:

Email:

\section{SUBMISSION INSTRUCTIONS}

Visit the SMJ website: http://www.smj.org.sg/current-issue and select the appropriate quiz. You will be redirected to the SMA login page.

For SMA member: (1) Log in with your username and password (if you do not know your password, please click on 'Forgot your password?'). (2) Select your answers for each quiz and click 'Submit'.

For non-SMA member: (1) Create an SMJ CME account, or log in with your SMJ CME username and password (for returning users). (2) Make payment of SGD 21.40 (inclusive of $7 \%$ GST) via PayPal to access this month's quizzes. (3) Select your answers for each quiz and click 'Submit'.

RESULTS:

(1) Answers will be published online in the SMJ February 2020 issue. (2) The MCR numbers of successful candidates will be posted online at the SMI website by 7 February 2020. (3) Passing mark is $60 \%$. No mark will be deducted for incorrect answers. (4) The SMJ editorial office will submit the list of successful candidates to the Singapore Medical Council. (5) One CME point is awarded for successful candidates. (6) SMC credits CME points according to the month of publication of the CME article (i.e. points awarded for a quiz published in the December 2019 issue will be credited for the month of December 2019, even if the deadline is in January 2020).

Deadline for submission (December 2019 SMJ 3B CME programme): 12 noon, 31 January 2020 